\title{
Searching for signals from the dark universe
}

\section{At the Italian Gran Sasso National Laboratory, a novel experiment that is in search of signs of the invisible dark matter that pervades our universe reports an intriguing result.}

\begin{abstract}
A large part of the dark matter that seems to dominate the universe is expected to be in the form of relic particles. This "dark universe" could be a unique window through which we will be able to look for new physics. The DAMA experiment at the INFN Gran Sasso National Laboratory is investigating this new frontier and sees an annual variation, which is suggestive of the Earth's motion against a background "wind" of particles.
\end{abstract}

\section{Particles from the dark universe}

Measurements of luminous matter (stars) lead to the conclusion that the universe does not contain enough stellar matter to halt the residual Big Bang expansion. Without enough such gravitational braking, the universe will continue to expand forever. However, many experimental observations suggest that luminous matter is not the end of the story. To account for the observed motion in the cosmos, gravitational fields much stronger than those attributable to luminous matter are required - more than about $90 \%$ of the mass in the universe should be the result of invisible dark matter.

This conclusion is further supported by simulations using cosmological models that point out the necessity for large numbers of relic particles - weakly interacting massive particles (WIMPs) - from the early universe.

This scenario implies that our galaxy should be completely embedded in a large WIMP halo. Our solar system, which is moving with a velocity of about $232 \mathrm{~km} / \mathrm{s}$ with respect to the galactic system, feels a continuous WIMP "wind".

The quantitative study of this wind would provide information on the evolution of the universe and investigate new physics possibilities. The lightest neutral particle (the neutralino) expected by the supersymmetric extension of the Standard Model is the best WIMP candidate.

\section{How to catch a WIMP?}

Direct detection of WIMPs is very difficult because they rarely interact. WIMP searches should be shielded from cosmic rays and operate in an environment of very low radioactivity. The detectors should be built using low-radioactive materials.

DAMA's home is deep underground in the INFN Gran Sasso National Laboratory in Italy. The collaboration (involving the University and INFN-Roma2, University and INFN-Roma, IHEP-

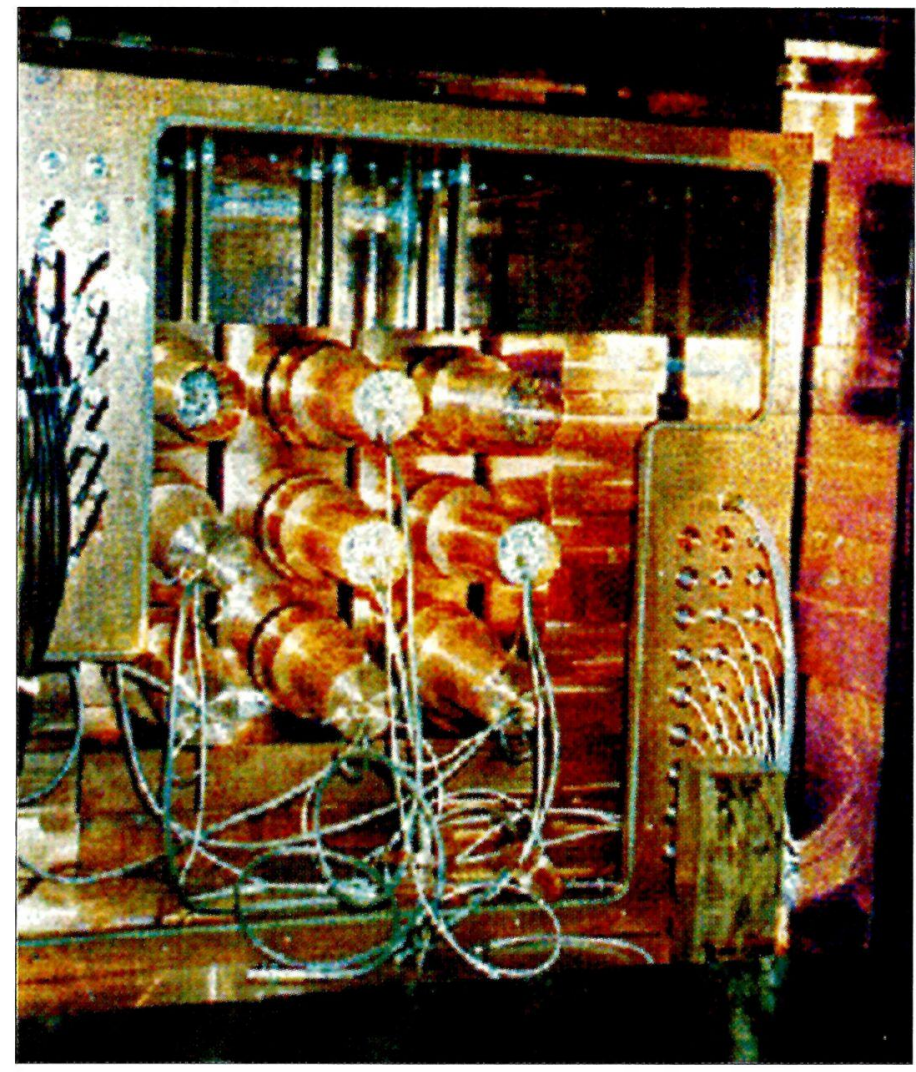

The DAMA experiment in the Italian Gran Sasso underground laboratory is searching for signs of WIMPS as a component of the invisible dark matter of the universe. The apparatus contains $100 \mathrm{~kg}$ of $\mathrm{Nal}(\mathrm{TI})$.

Beijing) is mainly devoted to the search for WIMPs in the same mass and cross-section region as accelerator experiments, and several results have already emerged.

Thus high-atomic-number target nuclei, such as iodine (in the form of $\mathrm{Nal}(\mathrm{TI})$ ) and xenon (in the form of a liquid xenon scintillator) are used. The search mainly focuses on WIMP-nucleus elastic scattering from the target-nuclei part of the detector, which would show up via nuclear recoil energies in the kilo-electronvolt range.

To help to isolate a possible WIMP signal from the background, the main feature of the WIMP wind is its annual modulation. As the Earth 


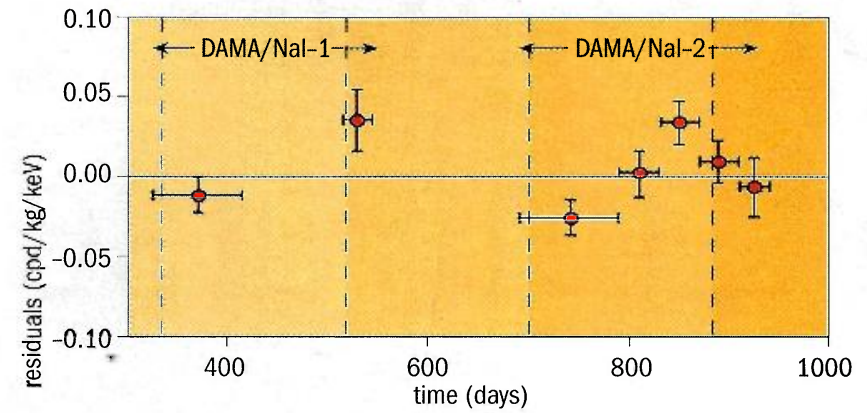

Fig. 1: The DAMA experiment sees an annual variation, suggestive of the effect of the Earth's motion against a background "wind" of dark matter particles. The modelindependent residual rate in the lowest energy interval seen by the DAMA experiment is shown as a function of the time elapsed since 1 January of the first year of data taking. The expected behaviour of a WIMP signal is a cosine function with the minimum roughly at the dashed vertical lines and with the maximum roughly at the dotted lines. The complete time and energy correlation data analysis is a similar modulation.

rotates around the Sun, it would be crossed by a higher WIMP flux in June (when its rotational velocity is in the same direction as that of the solar system with respect to the galaxy) and by a smaller one in December (when the two velocities are subtracted). The fractional difference of the rate is some $7 \%$.

To see such modulation requires heavy, stable detectors with appropriate features and stability control. The $100 \mathrm{~kg}$ highly radiopure Nal(TI) DAMA set-up is an example. Clear signatures overcome the difficulties of comparing different experiments and techniques.

\section{The $100 \mathrm{~kg}$ DAMA Nal(TI) set-up}

DAMA uses highly radiopure $\mathrm{Nal}(\mathrm{TI})$ scintillators that are produced in collaboration with the CRISMATEC company. All of the materials and the crystal growth and handling procedures have been studied carefully. A major effort has gone into optimizing the detectors and the electronics to give a relatively high number of photoelectrons per kilo-electronvolt and a low noise level.

The low background photomultiplier tubes employed in the experiment (two for each detector, working in coincidence at a single photoelectron threshold) have been developed by Electron Tubes Ltd. The materials were preselected by the company and their radioactivity was measured deep underground.

The other main parts of the experiment are the passive shield (to exclude environmental contributions to the counting rate), which surrounds the copper box housing the detectors, and the glovebox placed on the top of the shield for calibration. The whole of the apparatus is kept in highly pure nitrogen with slight overpressure with respect to the atmosphere in order to keep out radioactive radon gas.

The materials of the shield have been selected and monitored for low radioactivity. The upper glovebox is used to insert radioactive sources to calibrate the detectors in the same experimental conditions as those occurring during the production measurements.

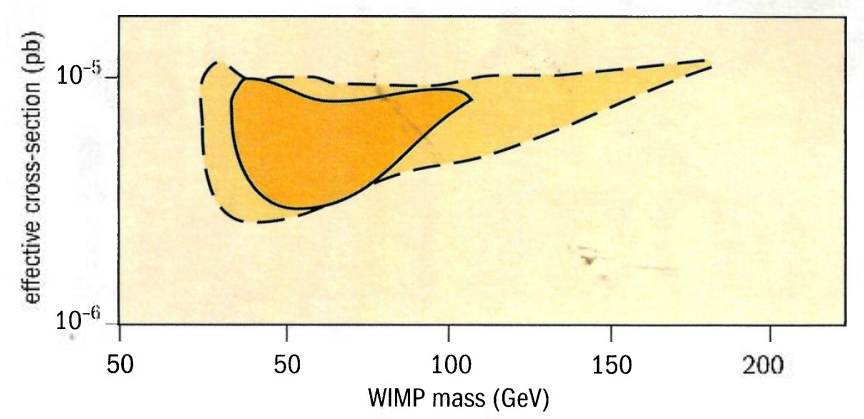

Fig. 2: DAMA sees a subtle variation in the annual count rate. Complying with many criteria (see text), it can be interpreted as being as a result of a relic neutralino. The vertical axis is the WIMP-nucleon cross-section multiplied by the WIMP local density in $0.3 \mathrm{GeV} / \mathrm{cm}^{3}$. The solid line contour is the combined analysis of the annual modulation DAMA data considering standard values for the local and escape velocities of the galaxy. The dashed line contour comes from varying these velocities around their mean values: between 170 and $270 \mathrm{~km} / \mathrm{s}$ for the local velocity and between 450 and $650 \mathrm{~km} / \mathrm{s}$ for the escape velocity.

This set-up is mainly devoted to studies of WIMP annual modulation, therefore particular care has been taken in the stability and monitoring of the running condition parameters, such as the operating temperature, the high-purity nitrogen flux, the glovebox overpressure, the total and single hardware rates above single photoelectron threshold, the environmental radon level and so on. All of the information related to these parameters is continuously recorded with actual data.

The experiment is taking data from a single photoelectron threshold to several mega-electronvolts, although the hardware conditions are obviously optimized for the lowest energy region. Pulse shape information is recorded over a period of 3250 ns for the lowest energy events.

\section{Searching for annual modulation}

Any annual modulation of the WIMP-nucleus differential energy spectrum should have all of the following features attributable to WIMP interactions:

- a modulation varying as a cosine function;

- a period of exactly one year;

- a proper summer-winter phase;

- only seen in a defined low-energy region;

- single "hit" events;

- a modulated amplitude below $7 \%$.

Single-hit events with only one detector firing are the ones of interest in WIMP search, the probability of a WIMP interacting in more than one detector being negligible.

After checking the monitored parameters, the time-dependent component of the rate is extracted from the collected data by grouping the events in cells of one day, $1 \mathrm{keV}$ and one detector. The number of events in each cell is then compared by applying a maximum 
likelihood analysis with the expectation from the standard WIMP model. The limit on the neutralino mass (the most favoured WIMP candidate) achieved at accelerators is taken into account.

The analysis of a first dataset suggested the possible presence of a signal compatible with the features of a neutralino with dominant spin-independent interaction. A second year of data taking with larger statistics has underlined this possibility. The signal modulation is shown in figure 1 and the neutralino interpretation in figure 2 .

The combined analysis of these two years of data (total statistics: $19511 \mathrm{~kg} /$ day) provides a confidence level of $99.6 \%$ for a neutralino mass of $59 \mathrm{GeV}(+17 /-14)$ and a proton cross-section of $7.0(+0.4 /-1.2) 10^{-6} \mathrm{pb}$ in the frame of the standard WIMP model.

Possible systematic effects and alternative explanations have been investigated, as discussed for example at the 3K Cosmology International Conference in Rome last October. None of the effects considered could simulate all six of the criteria for the annual modulation signature and provide the observed modulation.

Despite this the collaboration has been very cautious - mindful of the difficulties of dealing with rare events - and has increased its efforts to investigate all aspects of this intriguing result.

The region singled out by DAMA is consistent with the hypothesis of a relic neutralino as a dominant component of the cold dark matter in the galactic halo, as has been pointed out by a Turin group, which also says that some properties of the relevant supersymmet-

\section{Need to probe microstrip detectors or front-end VLSI's? \\ Maehlum Instruments PS150 simplifies and speeds up the work.}

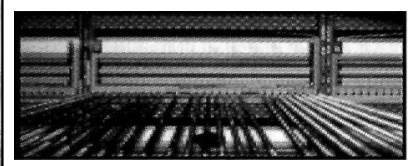

\section{Applications:}

Microstrip detectors front-end ASICS, materials research, Kapton interconnects, and so on.

\section{Key specifications:}

Ultra stable construction. 150x 150x $12 \mathrm{~mm}$ movement. $0.25 \mu \mathrm{m}$ resolution.

LabView drivers.

IEEE488, RS232 interface. Joy-stick manual control. $300 \mathrm{~mm}$ microscope travel. Accepts various microscopes. Accepts common probecards Custom designs possible.

\section{AS}

Maehlum Instruments AS $\begin{array}{ll}\text { phone: } & +4722580132 \\ \text { fax: } & +4722580133\end{array}$

www.maehlum.com info@maehlum.com ric particles should be accessible at present accelerators and in WIMP indirect searches.

The inclusion of these relic neutralinos in supergravity models hs also been considered by American physicists. A group from Rome and Moscow suggested that the effect could be the result of a heavy neutrino. Finally, the effect of the uncertainties on the dark halo local density and on the WIMP velocity distribution has been examined recently with the conclusion that the relic neutralinos possibly involved in the annual modulation effect would have a mass in the 30-130 GeV range with an upper bound extending to some $180 \mathrm{GeV}$ when possible bulk rotation of the dark matter halo is introduced.

\section{Perspectives and plans}

The analysis of further statistics is in progress as well as further data taking and an upgrade of the apparatus. If new research for the improved radiopurification of $\mathrm{Nal}(\mathrm{TI})$ is successful, the active mass could be increased to $250 \mathrm{~kg}$.

A final result would mean reproducing the effect over several annual cycles, including all tof he consistency checks.

- More information is available from "http://www.Ings.infn.it/ Ings/htexts/dama/".

\section{Rita Bernabei, Rome.}

Wide Band, Precision

\section{CLAMP ON CURRENT MONITORS}

Clamp-on versions of several Pearson ${ }^{\mathrm{TM}}$ Current Monitors are now available.

These incorporate wide-band frequency response in a demountable configuration for use on fixed conductor. Hole diameter is 2 inches, and sensitivity ranges from 0.001 to 1.0 Volt/Ampere.

Model 3525, typical of the group, has a sensitivity of $0.1 \mathrm{~V} / \mathrm{A}$, a frequency range of $5 \mathrm{~Hz}$ to $15 \mathrm{MHz}$, and 5,000 Amperes peak pulse current capability. Pulse rise-times down to 25 nanoseconds can be viewed.

We welcome inquires regarding custom clamp-on monitors to meet special requirements.

PEARSON ELECTRONICS, INC.

1860 Embarcadero Road

Palo Alto, California 94303, U.S.A.

Telephone (650) 494-6444 • FAX (650) 494-6716 Visit our web site at www.pearsonelectronics.com 